\section{Ultrasound assisted great saphenous vein ligation and division: an office procedure}

\author{
Stefano Ricci, Leo Moro, \\ Raffaele Antonelli Incalzi \\ Centro di Flebologia, Area di Geriatria, \\ Università Campus Bio Medico, Roma, \\ Italy
}

\section{Abstract}

The aim of this proof of concept study is to describe an ultrasound (US) assisted simplified surgical procedure for pre-terminal great saphenous vein (GSV) high ligation/division avoiding groin dissection and tributary interruption, in an office setting, in association to varices phlebectomy and saphenous vein foam occlusion treatment. Inclusion criteria: primary GSV reflux due to terminal valve, vein diameter $>6 \mathrm{~mm}$. By ultrasonography in standing position, the point GSV passing over the adductor longus muscle (about $3 \mathrm{~cm}$ from the junction) is identified. This $\mathrm{E}$ (easy) point, relatively superficial, free from tributaries and other structures, allows an easy grasping and extraction of the GSV vein through a $3 \mathrm{~mm}$ stab incision provided an ultrasonography assistance. The vein is divided/ligated about $2 \mathrm{~cm}$ distal from the ostium, the distal stump is cannulated and foam is injected on the distal segment from the E-point incision in a retrograde fashion, varices are avulsed by phlebectomy. Twenty procedures in 18 patients (venous clinical severity score: mean 3.15 - GSV diameter: mean 7.34) were performed, all the cases without inconveniences, with a duration not exceeding $10 \mathrm{~min}$ in addition to the phlebectomy procedure time. No complications as hemorrhage, infection, nerve lesion, lymphatic leak or thrombosis have been registered. At one month the residual saphenous stump length was in average $2.16 \mathrm{~cm}$ with complete closure of GSV in all. Three patients have been controlled at 6 months showing GSV complete closure. The procedure described is a simple office US assisted method for GSV ligationdivision, leaving the 2 last $\mathrm{cm}$ of the saphenofemoral junction. It could be associated to most of the procedures in use with limited additional time and resources required.

\section{Introduction}

Several methods are in use for achieving great saphenous vein (GSV) exclusion [high ligation, high ligation + stripping, endovascu- lar heat occlusion-laser/radio frequency (RF), chemical occlusion-sclerotherapy, glue occlusion-cyanoacrylate, steam occlusion, and others] in varicose veins treatment. Laser and RF, in particular, have achieved efficient GSV stem occlusion at 1 year (91\%-95\%) ${ }^{1}$ and even at 5 years long term follow up (73-86\%), $)^{2,3}$ with sparing of the proximal part $(2-3 \mathrm{~cm})$ of the GSV. Indeed, leaving this part patent and drained through the sapheno-femoral junction (SFJ) allows the saphenous tributaries to maintain their physiological drainage. In fact recurrence after surgery is frequently associated to groin neovascularisation which is possibly triggered by tributaries dissection/ligation, ${ }^{4}$ one of the proposed pathophysiological mechanisms. Avoiding both groin dissection and tributary interruption could explain the lower incidence of groin reflux recurrence referred by endovascular procedures, ${ }^{5,6}$ but confirmatory long term randomized studies are still lacking.

Furthermore, there is some evidence ${ }^{7}$ that a detailed ultrasound (US) SFJ investigation could avoid the need of dissection in almost the half of cases, while for the remaining ones a minimally invasive approach like the proposed one could lead to a decrease in the recurrence rate.

This view is at variance with the traditional belief that the junction need to be fully dissected and all the tributaries interrupted. ${ }^{8,9}$

Isolated GSV surgical high interruption, as an alternative to traditional saphenectomy can be useful in several selected conditions (summarized in Table 1). While treating varicose disease in an office setting, we conceived an US assisted simplified surgical procedure for high ligating/dividing the GSV, avoiding tributary interruption. This proof of concept study summarizes our initial experience with this method. It provides technical details of the method, the solutions we found to overcome some difficulties and the main indications.

\section{Materials and Methods}

\section{Patients}

Eighteen patients underwent 20 GSV preterminal interruptions (PTI) from October 2013 to April 2014 [12 female, 6 male; meanage: 46 (from 28 to 70); mean-body mass index: 24.23 (from 16.6 to 31.8]. Selection and exclusion criteria are reported in Table 2.

Candidates were selected from the patients scheduled for primary varicose vein treatment in our dedicated office based surgical activity (Ambulatorio Flebologico, Area di Geriatria, Campus Bio-Medico, Roma, Italy).

All patients were C2sEpAsPr ${ }_{2,3,5}$ of the clinical class of CEAP (Clinical-Etiology-AnatomicPathophysiologic) classification, ${ }^{10}$ symptomatic and with evident and extended bulging
Correspondence: Stefano Ricci, Corso Trieste 123, 00198 Roma, Italy.

Tel.: +39.327.5405566.

E-mail: varicci@tiscali.it

Key words: great saphenous vein ligation, ultrasound guidance, office procedure.

Contributions: SR, study design, data analysis, writing, final approval; LM, data collection, data analysis, critical review, final approval; RAI, data analysis, critical review, final approval.

Conflict of interests: the authors declare no potential conflict of interests.

Received for publication: 11 June 2014

Revision received: 29 July 2014.

Accepted for publication: 31 July 2014.

This work is licensed under a Creative Commons Attribution 3.0 License (by-nc 3.0).

(C) Copyright S. Ricci et al., 2014

Licensee PAGEPress, Italy

Veins and Lymphatics 2014; 3:4428

doi:10.4081/vl.2014.4428

varicosities with a GSV stem $>6 \mathrm{~mm}$ (reflux cutoff value $\geq 1 \mathrm{~s}$ ). In two cases the procedure was bilateral. Written informed consent was obtained from every patient.

\section{Methods}

In our practice varicose disease is treated by office based stab avulsion phlebectomy of varicosities, associated to US assisted foam sclerotherapy (4- $6 \mathrm{~mL}, 3 \%$ polidocanol 1:4 of air Tessari method ${ }^{11}$ ) of the saphenous stem when the SFJ is incompetent, provided that GSV diameter, measured - avoiding isolated dilatations - at $15 \mathrm{~cm}$ below the junction, exceeds 6 mm. ${ }^{12-14}$ For GSV $\leq 6 \mathrm{~mm}$ only the varicosities avulsion is made. Indeed, GSV treatment likely is not indicated due to possible residual efficiency of ostial/femoral valves. ${ }^{7,15}$ In the planning phase, the GSV is initially studied in standing position by echography during the preoperative veins marking phase. The vein is followed distally, starting from the junction, and the point passing over the adductor longus muscle (about $3 \mathrm{~cm}$ from the junction) is identified (Figure 1A).

This site, that we call the easy (E) point, the GSV stem is relatively free from other structures contact (collateral branches, lymph nodes, deep veins and arteries, nerves), lies over a muscular plane and is only covered by a thick, highly echogenic superficial fascia (Figure 1B). The overlying skin is marked at this point. Then, all the veins to be avulsed are marked on the skin and the patient is prepared for the procedure. Local infiltration anesthesia (mepivacain $0.4 \%$ solution prepared with 
bicarbonate $1.4 \%{ }^{16}$ is done over the markings but is made only superficially at the groin marked site to avoid hiding of the GSV by the subcutaneous infiltration. After varicosities phlebectomy is completed, US assistance is prepared at the groin using a probe sterile covering and sterile gel; a $3 \mathrm{~mm}$ transversal incision is made at marked site; by blunt dissection of the superficial subcutaneous tissue, a mosquito forceps is progressively advanced till the fascia layer over the GSV, observed in a transverse section. The fascia is repeatedly grasped by the forceps, possibly pulled out of the incision and sectioned to achieve a true opening of the structure: this will facilitate the vein's extraction. A right angle tiny forceps (Figure 2) is then advanced in the deeper space between the GSV and the adductor longus muscle and passed below the vein, under direct echo-visualization. Further anesthetic is then injected to obtain complete painless procedure, pushing the infiltration both towards the groin and over the distal part of the vein. (This will allow pulling the vein out in both directions). The GSV is then gently extracted from the skin incision (Figure 3A), freed from residual connective adhesions, sectioned between two forceps. The distal lower end is cannulated (Jelco Optiva ${ }^{\circledR}$ 2, I.V. Catheters, 20G; Smiths Medicals, St. Paul, MN, USA) and foam (4-6 mL) injected, until foam comes out of the distal phlebectomy stab incisions (for this reason performed previously), and ligated. The proximal upper end is gently pulled out and transfixed with $3 / 0$ Vycril suture without occluding the tributaries. Few drops of sclerosing agent are dripped in the residual part of the ligated stumps to eliminate all the endothelium remnants. The two ends are actively repositioned under the fascia layer, to avoid adhesions to the skin. The incision edges are approached by a steri-strip. A compressive adhesive bandage is applied on the entire limb starting from the groin, to be worn for a week. Patients are invited to walk immediately and actively during the following hours; no anticoagulation prophylaxis is prescribed unless in presence of hyper coagulation status.

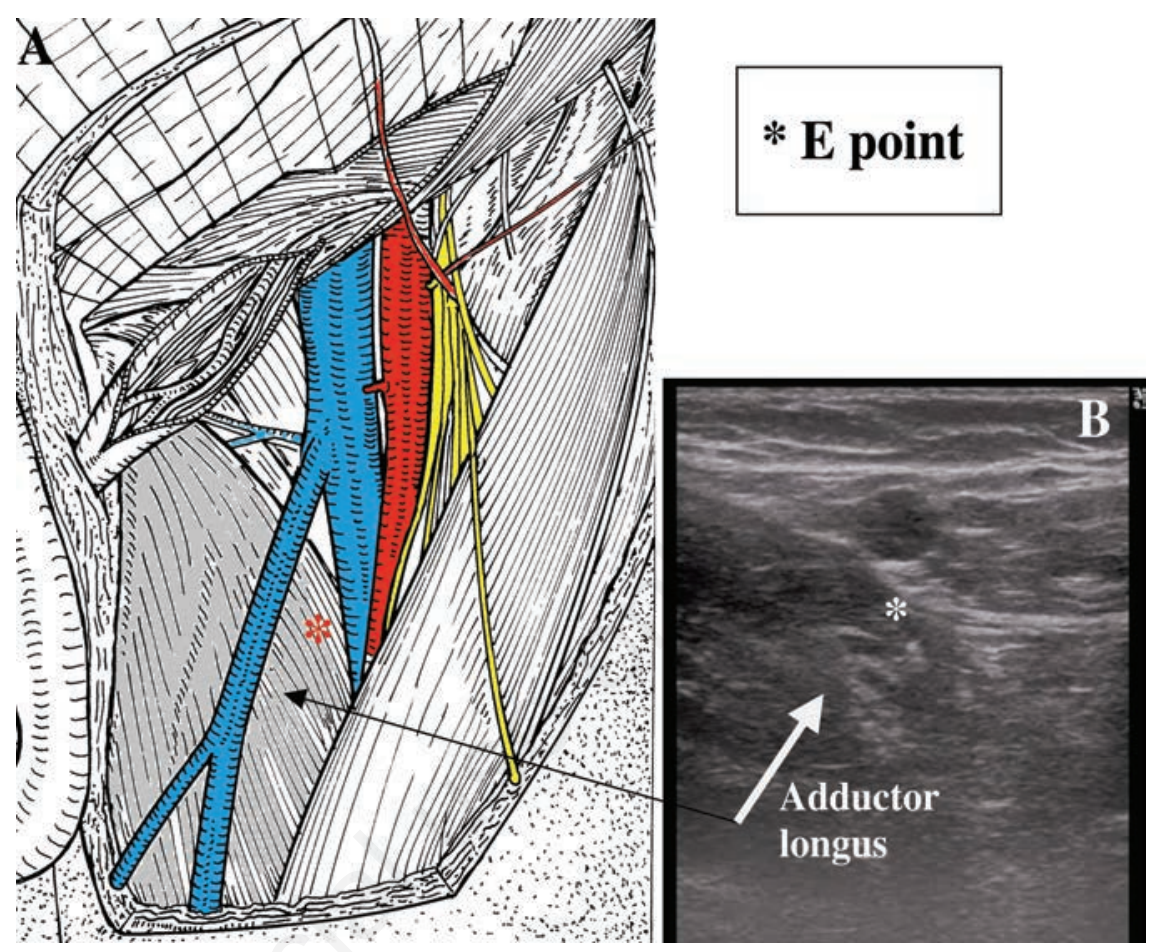

Figure 1. A) About three $\mathrm{cm}$ far from the ostium the great saphenous vein (GSV) lies over the adductor longus muscle, in a point $\left(\mathrm{E}\right.$ point $\left.{ }^{*}\right)$ free from collateral branches, lymph nodes, nerves, deep veins and arteries (modified from: Bardeleben $K H$, Haeckel E. Atlas of applied (topographical) human anatomy for students and practitioners. New York, NY: Rebman Company; 1906). B) At the E point (*) the GSV lies over the muscle plane and is covered by a well visible superficial fascial plane.

Table 1. Indications of sapheno-femoral junction isolated interruption.

Traditional saphenectomy not suitable (patient aged, co-morbidity, ulcers)

To obtain a more efficient sclerotherapy of the saphenous stem

When sclerotherapy has failed or is contra-indicated (PFO + neurologic symptoms, thrombophylia, allergy, non acceptance)

When a saphenous conservative method is preferred (CHIVA)

Ascending GSV thrombosis needing to be arrested or avoided

SFJ interruption associated to endovascular treatments

PFO, patent foramen ovale; CHIVA, conservative hemodynamic correction of venous insufficiency; GSV, great saphenous vein; SFJ, sapheno-femoral junction.

Table 2. Inclusion and exclusion criteria.

Inclusion criteria

Primary GSV reflux due to terminal valve incompetence (Valsalva and compression/release positive over the junction, reflux $>1 \mathrm{~s}$, in standing position)

Vein diameter $>6 \mathrm{~mm}$ measured at $15 \mathrm{~cm}$ distal from the groin

Length of the incompetence $>30 \mathrm{~cm}$ (continuous, non interrupted)

Competent junction tributaries

Exclusion criteria

Clinical state not suitable for office surgery

Depth of GSV (skin-vein US distance) $>3 \mathrm{~cm}$

Previous GSV sclerotherapy

GSV, great saphenous vein; US, ultrasound. 
Clinical and Duplex monitoring for these first cases are scheduled at 7 and 30 days, and then at 6 and 12 months.

\section{Results}

Twenty cases in 18 patients have so far been operated. The mean venous clinical severity score $^{17}$ was 3.15 [from 1 to 5 ; standard deviation (SD): 1.04]. The mean-diameter of GSV at $15 \mathrm{~cm}$ distal from the groin was $7.34 \mathrm{~mm}$ (6$10.5 \mathrm{~mm}$; SD: 1.12). The duration of the specif- ic procedure, as part of a phlebectomy session (lasting in average $45 \mathrm{~min}$ ), has never exceeded $10 \mathrm{~min}$. In no case the procedure has been interrupted. No complications as hemorrhage, infection, nerve lesion, lymphatic leak or thrombosis have been registered; one patient presented an asymptomatic edema around the groin incision lasting 15 days, treated by compression and antibiotics. Incision healing resulted very satisfactory (Figure $3 \mathrm{~B}$ ). In one case skin retraction at the scar was registered, in treatment with massaging.

All the patients have been clinically and US controlled at 7 days and 30 days. At one month the residual saphenous stump length was in

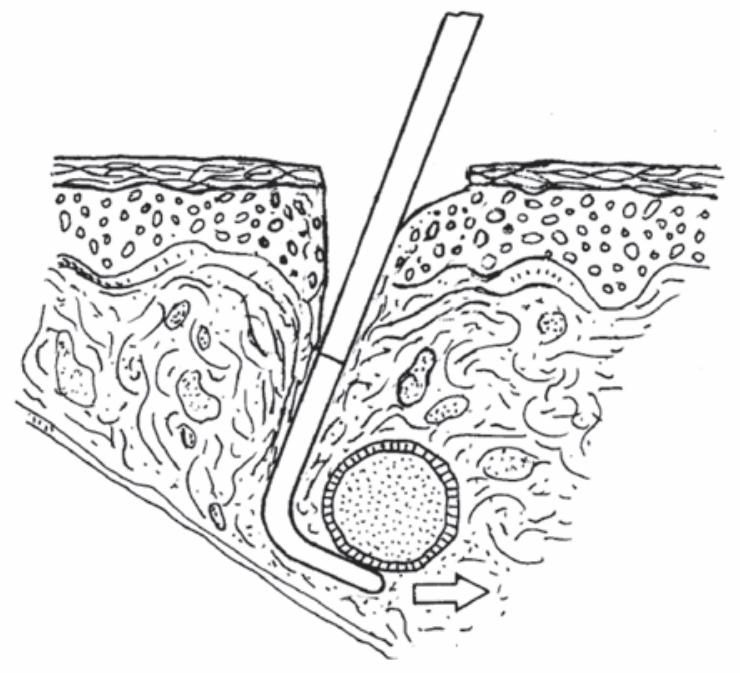

Figure 2. Through a fascia opening a right angle tiny forceps is advanced in the deeper space between the great saphenous vein and the adductor longus muscle and passed below the vein, under direct echo-visualization. average $2.16 \mathrm{~cm}$ (1.18-3; SD: 0.52) with complete closure of GSV in all. In all the cases the stump was patent without reflux. Eight patients have been controlled at 6 months showing GSV complete closure; one case presents at Valsalva a stump filling due to a pelvic reflux (Table 3 ) feeding the stump through a pudendal vein (not to a reflux from the ostium).

\section{Discussion}

This proof of concept study suggests that the proposed GSV PTI is easy to be performed and safe. It lends support to the current propensity to spare the last $2 \mathrm{~cm}$ of the GSV, to allow SFJ tributaries physiological drainage. It is effective at short term when associated to foam sclerotherapy, due to the wash out effect elim-

Table 3. Demographic, clinical characteristics and results of participants.

Demographic and clinical characteristics

All procedures, no.

Patients, no.

Gender, no. (\% women)

Age mean

BMI mean; DS

GSV vein diameter at $15 \mathrm{~cm}$ distal from the groin mean (DS); $\mathrm{mm}$

VCSS mean; DS

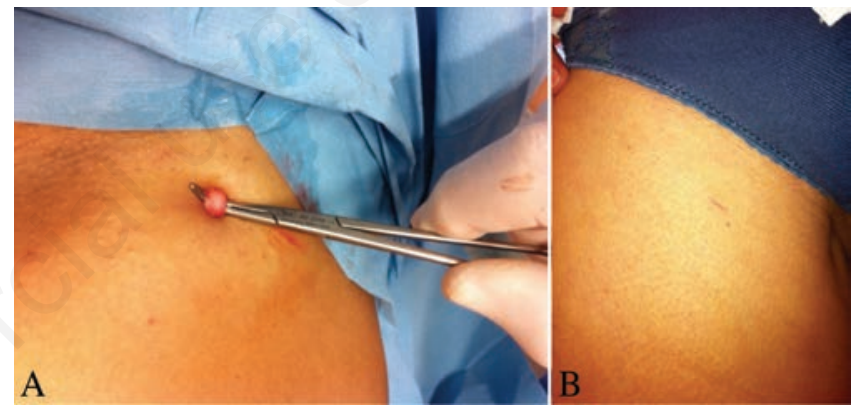

Figure 3. A) The great saphenous vein extracted from the skin incision will be divided and ligated. B) At one month the incision healing is usually very satisfactory.

Result:

Saphenous stump length mean (DS); cm

Patent stumps, no.

Reflux by Valsalva maneuver, no.

20

18

$2(66.6)$

$46(28-70)$

$24.23 ; 3.99$

$7.34(6-10.5) ; 1.12$

$3.15(1-5) ; 1.04$

Results at 6 months ( 8 controls)

Saphenous stump length mean (DS); cm

$1.65(1-2.5) ; 0.52$

Patent stumps, no.

8

Reflux by Valsalva maneuver, no.

1 (pudendal)

BMI, body mass index; SD, standard deviation; GSV, great saphenous vein; VCSS, venous clinical severity score. 
ination. Longer outcome evaluation will need considering possible bias due to the state of the related superficial vein network (possible recurrence, perforators hemodynamic, phlebectomy outcome).

Indeed, traditional flash to femoral vein high ligation has been universally considered as the gold standard operation to achieve GSV interruption. ${ }^{18}$ However, suspect is rising that junction recanalisation due to neo-vascularisation could be secondary to junction wide dissection ${ }^{19}$ and tributary interruption. ${ }^{5}$ Indeed, several Authors performing saphenectomies without junction dissection reported favorable outcome, with apparently better results compared to traditional junction dissection..$^{20-22}$ Endovascular venous closure treatments meanwhile show that GSV closure leaving the junction's last $1-2 \mathrm{~cm}$ open to allow tributaries physiological drainage is associated with limited recurrence rates. ${ }^{1,23-26}$

Although GSV high ligation seems to be unnecessary when associated to modern procedures, still GSV interruption could be useful in some specific clinical and anatomical situations other than traditional GSV stripping (Table 1).

However, traditional groin dissection is a true surgical procedure, even when performed in local anesthesia, requiring surgical skill and a protected, dedicated setting. For this reason a simple technique of GSV interruption like the one we suggest could fit all described situations, but with a limited cost, limited surgical experience required, good efficacy and safety. This technique being pre-terminal, i.e. sparing the junction tributaries and avoiding dissection, it could create lesser stimuli to neo-vascularisation, provided that the neovascularization reaction theory will be definitively confirmed. ${ }^{5,6}$ The residual $2-3 \mathrm{~cm}$ long saphenous stamp does not substantially differ from that left in place by the endovascular techniques.

About $15 \%$ of the saphenous stems treated by sclerotherapy may recanalise at 1 year follow up, ${ }^{27}$ up to about $50 \%$ at 5 years, ${ }^{1}$ requiring further foam sclerotherapy. Trying to avoid recanalisation we began to perform pre terminal GSV ligation/division during the same phlebectomy/sclerotherapy session to obtain a more complete and durable foam sclerosis. Our 6 months control shows no recanalization, but the numbers are limited and long-term patency needs to be evaluated.

Our GSV PTI takes inspiration from the Dortu's experience dating back to $1993^{28}$ followed by Fays-Bouchon in $1995^{29}$ and recently resumed by others ${ }^{20,21,30}$ suggesting a stab avulsion method of the saphenous stem with preterminal junction ligation. The GSV at the groin was found by pulling the distal GSV progressively extracted and feeling the saphenous stem under the skin ${ }^{28}$ or a stripper previously introduced in a distal approach. ${ }^{29}$ In our expe- rience, the modern habit of US observation revealed that the GSV becomes more superficial at 2-3 cm from the SFJ, when passing over the Adductor longus muscle. In that site, the $\mathrm{E}$ point, the vein has its medial side in contact with the muscular fascia, while its lateral side is free from any other dangerous structure, allowing an easy blunt dissection guided by US imaging. Interestingly, this part of the GSV is usually free from tributaries and is easily pulled out for $1-2 \mathrm{~cm}$ due to its elasticity.

Two recently published papers ${ }^{31,32}$ describe a GSV interruption technique by passing a tread below the GSV by US assistance, as a complement of endovenous laser ablation, with the purpose of avoiding risk of thrombosis and recurrence keeping the thermal energy away from the saphenofemoral junction. These authors simply apply a tread to close the vein in association to the Laser treatment.

The method that we suggest, at the opposite, allows GSV interruption, possible vein resection, vein cannulation for foam or endovenous ablation. Furthermore, a better visualization of the vein is accomplished avoiding the risk of femoral vein involvement. ${ }^{32}$

Finally, GSV PTI could perfectly fit the office based CHIVA (conservative hemodynamic correction of venous insufficiency) protoco ${ }^{33}$ or similar ${ }^{34}$ where the GSV interruption is done in the perspective of GSV stem sparing strategy. Compared to the other GSV interruption options (laser, RF, steam, glue) the PTI technique seems simpler, cheaper (no technology is needed) and, in particular, more precise in terms of anatomical positioning.

Attention should be drawn to assess the competence of the junction tributaries (particularly the anterior accessory SV) destined to drain in the residual GSV part. In case of one or more tributary incompetence, a reflux of the SFJ would be maintained active by the reverse flow in the same tributary, with possible fast recurrence. At the opposite, reflux absence in the residual stump is assured by the tributary valve competence. Finally, attention to the Giacomini vein (GV) hemodynamic should always be deserved, avoiding GSV proximal interruption when GV is the prevalent drainage of the small saphenous vein.

\section{Limitations}

Some limitations of the described method should be underlined: obese patients may be particularly cumbersome, especially if groin anatomy is unfavorable (deep skin creases); previous sclerotherapy may cause fibrous adhesions and GSV fragility; GSV diameter dilatation or aneurysm at the E Point may suggest more traditional surgical techniques. Finally, a good US experience is needed in the preoperative and operative phase.

Some technical points also will need to be analyzed in the future studies: should we shorten the proximal/distal stump surgically in order to make the neovascularization less probable? Should we do a simple ligature/titanium clip positioning on the GSV?

\section{Conclusions}

GSV PTI is a simple office surgical technique allowing a GSV ligation-division leaving the 2 last $\mathrm{cm}$ of the SFJ, possible thanks to US assistance. It could be associated to most of the procedures in use, if definite pre-terminal GSV interruption is wished, with limited additional time and resources required. However, only a long-term follow up will definitively prove its efficacy.

\section{References}

1. Rasmussen LH, Lawaetz M, Bjoern L, et al. Randomized clinical trial comparing endovenous laser ablation, radiofrequency ablation, foam sclerotherapy and surgical stripping for great saphenous varicose veins. Br J Surg 2011;98:1079-87.

2. Nicolini P; Closure Group. Treatment of primary varicose veins by endovenous obliteration with the VNUS closure system: results of a prospective multicentre study. Eur J Vasc Endovasc Surg 2005;29: 433-9.

3. Winterborn RJ, Corbett CR. Treatment of varicose veins: the present and the future: a questionnaire survey. Ann R Coll Surg Engl 2008;90:561-4.

4. Disselhoff BC, der Kinderen DJ, Kelder JC, Moll FL. 5-year results of a randomised clinical trial comparing endovenous laser with SFL ligation and stripping for great saphenous varicose veins. Br J Surg 2011;98:1107-11.

5. ChandlerJ G, Pichot O, Sessa C, et al. Defining the role of extended saphenofemoral junction ligation: a prospective comparative study. J Vasc Surg 2000;32: 941-53.

6. Theivacumar NS, Darwood R, Gough MJ. Neovascularisation and recurrence 2 years after varicose vein treatment for sapheno femoral and great saphenous vein reflux: a comparison of surgery and endovenous laser ablation. Eur J Vasc Endovasc Surg 2009;38:203e7.

7. Zamboni P, Gianesini S, Menegatti E, et al. Varicose vein surgery without saphenofemoral junction disconnection. Br $\mathrm{J}$ Surg 2010;97:820-5.

8. Bradbury AW. Varicose veins. In: Beard HD, Gaines PA, eds. Vascular and endovas- 
cular surgery. 2nd ed. London, UK: W.B. Saunders; 2001. pp 498-513.

9. Blomgren L, Johansson G, DahlbergAkerman A, et al. Recurrent varicose veins: incidence, risk factors and groin anatomy. Eur J Vasc Endovasc Surg 2004;27:269-74.

10. Bergan JJ, Eklof B, Kistner RL, et al. Classification and grading of chronic venous disease in the lower limbs. A consensus statement. Vasc Surg 1996;30:5-11.

11. Tessari L. Nouvelle technique d'obtention de la scléromousse. Phlebologie 2000;53: 129 .

12. Mendoza E, Blättler W, Amsler F. Great saphenous vein diameter at the saphenofemoral junction and proximal thigh as parameters of venous disease class. Eur J Vasc Endovasc Surg 2013;45:76-83.

13. Mendoza E. Diameter reduction of the great saphenous vein and the common femoral vein after CHIVA Long-term results. Phlebologie 2013;42:65-9.

14. Cappelli M, Molino Lova R, Ermini S, et al. Hemodynamics of the sapheno-femoral complex: an operational diagnosis of proximal femoral valve function. Int Angiol 2006;25:356-60.

15. Cappelli M, Molino Lova R, Ermini S, Zamboni P. Hemodynamics of the sapheno-femoral junction. Patterns of reflux and their clinical implications. Int Angiol 2004;23:25-8.

16. Moro L, Serino FM, Ricci S, et al. Dilution of a mepivacaine-adrenaline solution in isotonic sodium bicarbonate, for reducing subcutaneous infiltration pain in ambulatory phlebectomy procedures: a randomized, double blind, controlled trial. J Am Acad Dermatol 2014 [In press].

17. Rutherford RB, Padberg FT Jr, Comerota
$\mathrm{AJ}$, et al. Venous severity scoring: an adjunct to venous outcome assessment. J Vasc Surg 2000;31:1307-12.

18. Bergan JJ. Surgery of the veins of the lower extremity. Philadelphia: WB Saunders; 1985.

19. Glass GM. Neovascularization in recurrence of the varicose great saphenous vein following transaction. Phlebology 1987; 2:81-91.

20. Pittaluga P, Chastanet S, Guex J-J. Great saphenous vein stripping with preservation of sapheno-femoral confluence: Hemodynamic and clinical results. MDJ Vasc Surg 2008;47:1300-5.

21. Casoni P, Lefebvre-Vilardebo M, Villa F, Corona P. Great saphenous vein surgery without high ligation of the saphenofemoral junction J Vasc Surg 2013;58: 173-8.

22. Mariani F, Bucalossi M, Mancini S, Mancini S. Selective vs radical crossectomy of the great saphenous vein in primitive venous insufficiency: results at 5 years. Acta Phlebologica 2009;10:5-10.

23. Pronk P, Gauw SA, Mooij MC, et al. Randomized controlled trial comparing saphenofemoral ligation and stripping of the great saphenous vein with endovenous laser ablation ( $980 \mathrm{~nm}$ ) using local tumescent anaesthesia: one year results. Eur J Vasc Endovasc Surg 2010;40:649-56.

24. Min RJ, Khilnani N, Zimmet SE. Endovenous laser treatment of saphenous vein reflux: long-term results. J Vasc Interv Radiol 2003;14: 991-6.

25. Ravi R, Trayler EA, Barrett DA, Diethrich EB. Endovenous thermal ablation of superficial venous insufficiency of the lower extremity: single-center experience with 3000 limbs in a 7-year period. J Endovasc
Ther 2009;16:500-5.

26. Merchant RF, Pichot 0; Closure Study Group. Long-term outcomes of endovenous radiofrequency obliteration of saphenous reflux as a treatment for superficial venous insufficiency. J Vasc Surg 2005;42: 502-9.

27. Winterborn RJ, Corbett CR. Treatment of varicose veins: the present and the future:a questionnaire survey. Ann R Coll Surg Engl 2008;90:561-4.

28. Dortu J. La crossectomie sus-fasciale au corse de la Phlebectomie Ambulatoire du complexe saphenien interne à la cuisse. Phlébologie 1993;46:123-36.

29. Fays-Bouchon N, Fays J. Une technique d'endo-eveinage de la saphéne interne en ambulatoire par micro-incisions. Phlébologie 1995;48:353-8.

30. Horakova MA, Horakova E. Ambulatory phlebectomy of incompetent great saphenous vein without flush saphenofemoral ligature: effect on the saphenofemoral junction. Phlebologie 2002;4:PAGES?

31. Okazaki Y, Orihashi K. Less Invasive ultrasonography-guided high ligation of great saphenous vein in endovenous laser ablation. Ann Vasc Dis 2013;6:221-5.

32. Zhu H-P, Zhou Y-L, Zhang X, et al. Combined endovenous laser therapy and pinhole high ligation in the treatment of symptomatic great saphenous. Ann Vasc Surg 2014;28:301-5.

33. Passariello F, Ermini S, Cappelli M, et al. The office based CHIVA. J Vasc Diagn 2013;2013:13.

34. Gianesini S, Menegatti E, Zuolo M, et al. Short endovenous laser ablation of the great saphenous vein in a modified CHIVA strategy. Veins and Lymphatics 2013;2:e21. 